\title{
Dielectric Permittivity in Paraelectric/Ferroelectric Coexistence Region in Several Proton Glasses
}

\author{
F. L. Howell, I. L. Fundaun, S. Stadler \\ Dept. of Physics \\ University of North Dakota, Grand Forks, ND 58202 \\ S. C. Meschia, C. S. Tu, V. H. Schmidt \\ Dept. of Physics \\ Montana State University, Bozeman, MT 59717
}

Abstract We present results for the complex permittivities of several proton glasses of the form $\mathrm{M}_{1-\mathrm{x}}\left(\mathrm{NZ}_{4}\right) \mathrm{x} \mathrm{Z}_{2} \mathrm{AO}_{4}$, where $\mathrm{M}=\mathrm{Rb}, \mathrm{Z}=\mathrm{H}$ or $\mathrm{D}$, and $\mathrm{A}=\mathrm{As}$ or $\mathrm{P}$. All measurements were made perpendicular to the ferroelectric axis, so no effects of domain wall motion occurred. Phase coexistence was apparent in all species. The phosphate glasses exhibited a much narrower coexistence composition range than the arsenate glasses.

\section{INTRODUCTION}

$\mathrm{RbH}_{2} \mathrm{PO}_{4}$ and corresponding potassium, cesium and arsenate compounds are ferroelectric, whereas $\mathrm{NH}_{4} \mathrm{H}_{2} \mathrm{PO}_{4}$ (ADP) and $\mathrm{NH}_{4} \mathrm{H}_{2} \mathrm{AsO}_{4}$ (ADA) are antiferroelectric. Mixed crystals of various concentrations of the ferroelectric and antiferroelectric components can be grown. Over a range of concentrations the response of these crystals is frustrated and no phase transition is exhibited. However, a range exists where either ferroelectric or antiferroelectric components can coexist with other dielectric phases. These crystals are, in many respects, the electrical analogs of magnetic "spin glasses," with pseudospin representing the two off-center hydrogen positions in the hydrogen bond. Unlike spin glasses and structural glasses, however, proton glasses possess random dc bias "fields" acting on these hydrogens. In proton glasses, there exists an ergodic limit below which the response to an external electric field exhibits thermal hysteresis [1].

Dielectric responses seen in these glasses are frequently modeled by lumped circuit elements or by multiple barriers. In any multiple barrier theory the height of at least some of the potential energy barriers must be posited to be as large as the thermal energy where the onset of nonlinearity occurs [2].

\section{EXPERIMENTAL PROCEDURE}

Two types of capacitance measurement devices were used in the data collection process. The General Radio Precision Measurement Bridge is a manual bridge that was used for $c$-axis measurements and the low temperature b-axis measurements. A digital bridge, Model 6425 Wayne-Kerr Component Analyzer, was employed for confirmation of b-axis measurements and measurement of the deuterated crystals. The General Radio Model consisted of a GR 1616 Precision Capacitance Bridge and a GR 1621 Capacitance Measurement System, consisting of a GR 1316 Oscillator and a GR 1238 Detector. After warm-up the accuracy is within \pm 1 percent. Measurements were taken by balancing the inphase and quadrature readings on the detector.

The ratio arms of the capacitance bridge are transformer windings. Fixed-capacitance standards are used whose values range in decade steps from $1 \mathrm{aF}$ to $100 \mathrm{nF}$. Loss in the measured capacitor is expressed as parallel conductance from the resolution limit of $0.1 \mathrm{f} \Omega$ to a maximum of $1 \mathrm{~m} \Omega$. The values of the set of five conductance standards are effectively extended by a series of resistance standards covering six decades. The Wayne-Kerr component analyzer has a direct readout of the sample capacitance and conductance and can be controlled by a computer via a general purpose interface bus (GPIB/HPIB).

All samples were cooled slowly in liquid nitrogen to $77 \mathrm{~K}$. They were then allowed to warm slowly to room temperature. Data were taken during the warming process. A thermocouple was embedded in teflon that fit tightly within a copper cylinder which was suspended less than $1 \mathrm{~mm}$ from the sample. The lower stem of the probe was wound with resistive wire and covered with a layer of plaster. A temperature controller regulated the amount of current flowing through the resistive wire. The sample probe was placed into a dewar and the lower portion covered with sand. Then the dewar was sealed with tape and styrofoam.

Measurements below $77 \mathrm{~K}$ were taken in a Princeton Applied Research Model 157 liquid helium cryostat. The rate of helium flow was controlled manually and temperature readings were taken with two LakeShore sensors: a CGR-2 carbon glass resistor and DT-500 diode. The CGR-2 was calibrated using superconducting transitions of various elements: lead, antimony, niobium and indium. The low-temperature probe held the sample by means of spring-loaded leads that "sandwiched" the sample into a position proximal to the heater and temperature measurement component.

\section{RESULTS AND DISCUSSION}

Two crystals of $\mathrm{Rb}_{1-\mathrm{x}}\left(\mathrm{NH}_{4}\right) \mathrm{H}_{2} \mathrm{PO}_{4}$ (RADP) were grown in order to determine the shape of the phase diagram at low concentrations of ammonium. The concentrations of ammonium in the crystals, $x=0.08$ and $x=0.16$, were assumed to resemble those of the solutions from which they were grown since (1) phase diagram results obtained using these concentrations agree qualitatively with previous results and (2). credible chemical analysis could not be obtained. The phase boundaries between ferroelectric (FE), paraelectric (PE), proton glass (PG) and the coexistent phases (F-P and F-G) are mapped in Fig. 1 as a function of temperature and concentration of ammonium $\mathrm{x}$ in the mixed crystals. This is the first report of coexistence of the ferroelectric phase with the paraelectric/proton glass phase in RADP. In an $x=0.75$ crystal, a plateau preceded and followed by abrupt decreases in the derivative of the real part of the perrnittivity with decreasing temperature, together with the onset of dispersion in the imaginary part of the permittivity at the occurrence of the lowertemperature change in the derivative of the real part of the permittivity [4], indicate the existence of an antiferroelectricproton glass coexistence region, although the authors at that time interpreted these phenomena as "reentrant behavior".

Dielectric measurements were made along the $c$ (ferroelectric) and $b$ axes of the undeuterated crystals. The real part of the dielectric permittivity very closely follows a Curie-Weiss behavior $\varepsilon^{\prime}=C /(T$ $\left.T_{0}\right)+\varepsilon_{1}$ above the temperature $T_{c}$ where ferroelectric behavior begins to occur, but differs substantially below $T_{c}$. Here $\varepsilon_{1}$ is the residual permittivity from the electronic and fast ionic response. The free parameters $\mathrm{C}$ and $\mathrm{T}_{0}$, obtained for rubidium dihydrogen phosphate (RDP), were within one percent of the published values [5]. The mixed crystal 


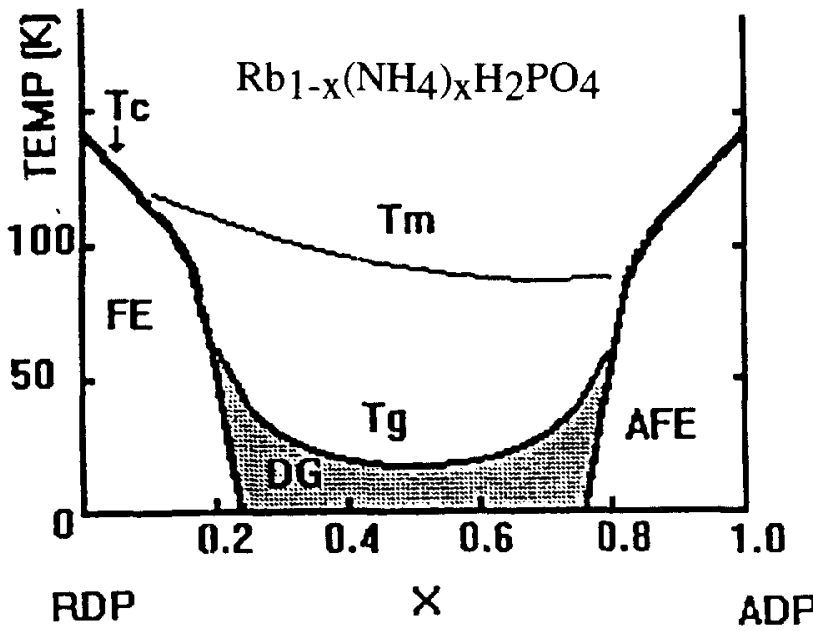

Fig. 1. Phase diagram for $\mathrm{Rb}_{1-x}\left(\mathrm{NH}_{4}\right)_{x} \mathrm{H}_{2} \mathrm{PO}_{4}$ [3]. FE represents the ferroelectric phase, AFE represents the antiferroelectric phase and DG represents the proton/dipole glass phase. The mixed crystal exhibits complete Curie-Weiss behavior above $T_{m}$.

with sixteen percent ammonium substituted for rubidium (RADP $\mathrm{x}=0.16$ ) yielded a Curie-Weiss parameter $T_{0}$ equal to $90.4 \mathrm{~K}$ and a Curie constant $\mathrm{C}$ of $5537 \mathrm{~K}$. The RADP crystal having eight percent ammonium yielded a $\mathrm{T}_{0}$ of $117 \mathrm{~K}$ and a Curie constant of $3203 \mathrm{~K}$. Knowing that the transitions in this region are ferroelectric, the results from the dielectric measurements could be added to those of previously published phase diagrams of concentration versus temperature [6]. The results so obtained agree qualitatively with the published phase diagrams.

Deuterated crystals were also grown from solutions with ammonium concentrations of 18 and 24 percent. As before, the concentration of ammonium in the crystal was assumed to be the same as that in the solution. The temperatures corresponding to the peaks in the imaginary part of the complex relative dielectric constant were assumed to follow a purely Arrhenius Law. By fitting to an Arrhenius behavior, an activation energy of $131 \mathrm{meV}$ is obtained for deuterated RADP (d-RADP). By contrast the activation energy of the undeuterated crystal RADP $(x=0.16)$ is only $4.14 \mathrm{meV}$ [7]. Phase data for d-RADP were obtained as before and added to previously published phase diagrams as shown in Fig. 2.

In addition, a crystal of RADA of 0.01 ammonium concentration was grown. The phase diagram containing this data point is shown in Fig. 3 for comparison with the phase diagrams of the related phosphate crystals. Note, however, that there is recent evidence from nuclear quadrupole resonance that the ferroelectricparaelectric coexistence region extends as low as $\mathrm{x}=0.01$, not 0.04 as shown in Fig. 3 [8].

\section{CONCLUSION}

This study continues research into the disordered phase in $\mathrm{Rb}_{1-\mathrm{x}}\left(\mathrm{NH}_{4}\right)_{\mathrm{X}} \mathrm{H}_{2} \mathrm{PO}_{4}$ (RADP). A ferroelectric transition and glassy freezing were observed in the RADP $x=0.16$ crystal at 90.5 $\mathrm{K}$ and $20 \mathrm{~K}$, respectively. A coexistence region occurs for RADP and from this it is suggested that the phase diagram for RADP should be modified to include a coexistence region from $x=0.22$ to $\mathrm{x}=0.16$. This modified phase diagram resembles the phase diagrams for deuterated RADP (d-RADP) and RADA.

The activation energy for RADP $x=0.16$ was anomalously low compared to those of $\mathrm{d}-\mathrm{RADP} \mathrm{x}=0.18$ and RADA $\mathrm{x}=0.10$. In addition, the real part of the residual dielectric permittivity of this sample is anomalously large at $2 \mathrm{~K}$ and continues to decrease with decreasing temperature [9]. Both of these anomalies may be associated with phonon-assisted tunneling [10].

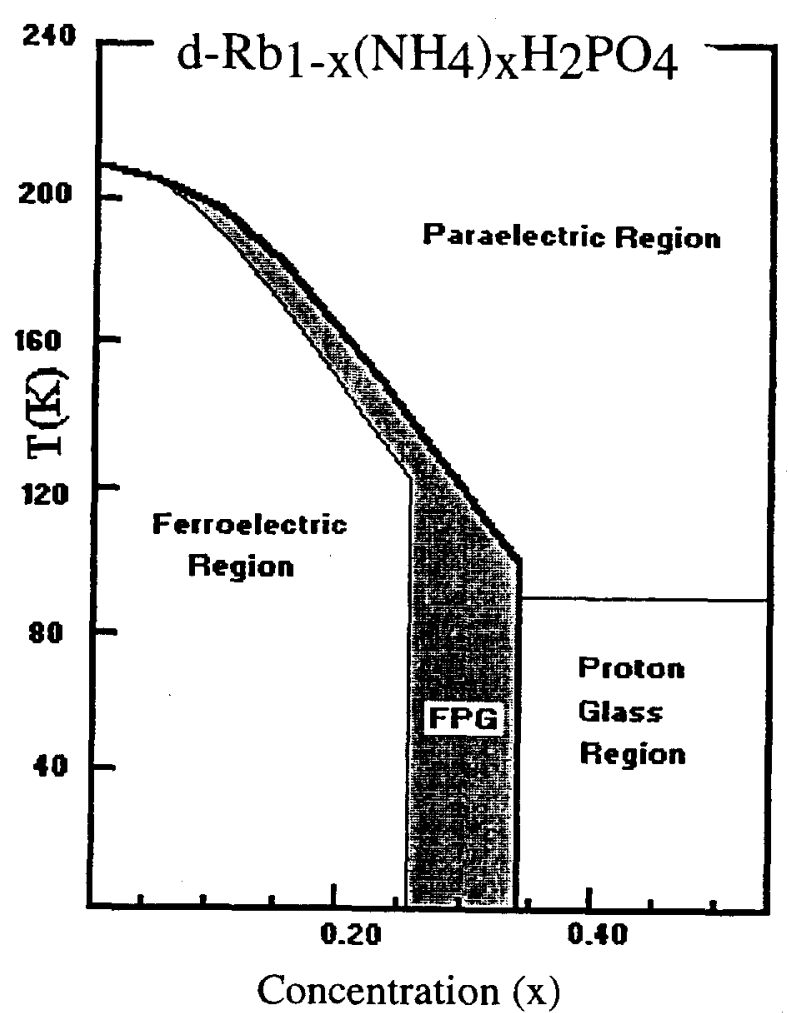

Fig. 2. Phase boundaries for $\mathrm{Rb}_{1-x}\left(\mathrm{ND}_{4}\right)_{\mathrm{x}} \mathrm{D}_{2} \mathrm{PO}_{4}$ ( $\mathrm{D}=$ deuterium) in the temperature-ammonium concentration plane. FPG represents a region of mixed ferroelectric and proton glass phases.

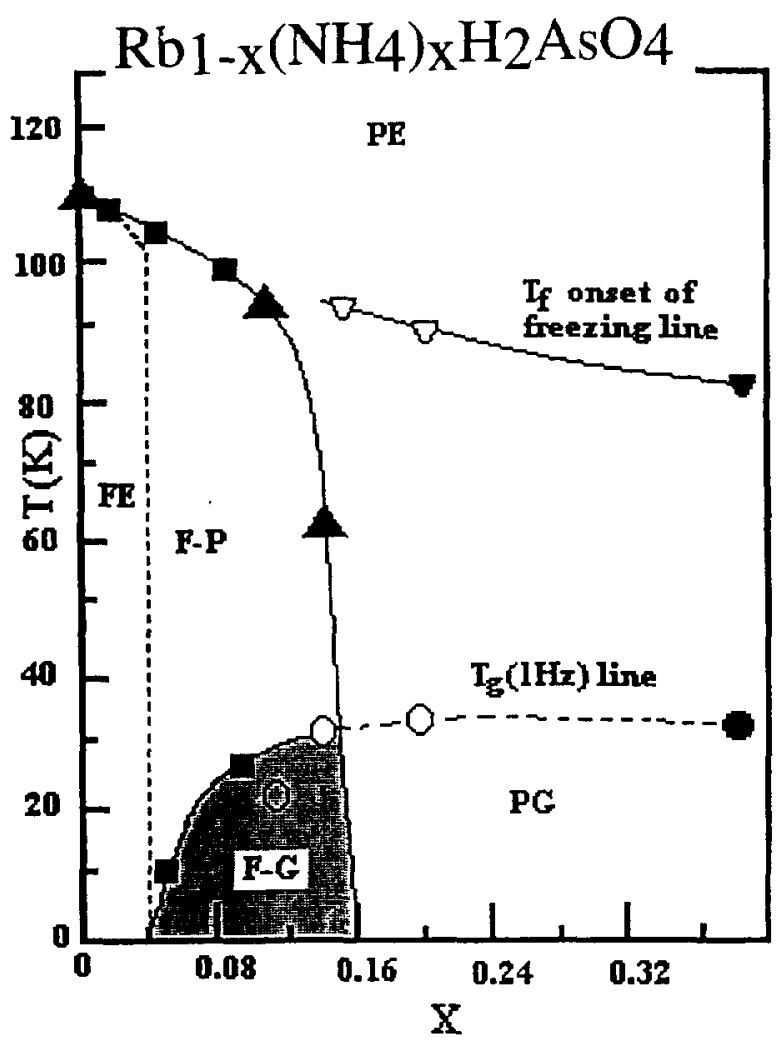

Fig. 3. Phase boundaries of $\mathrm{Rb}_{1-x}\left(\mathrm{NH}_{4}\right)_{\mathrm{X}_{2}} \mathrm{AsO}_{4}$ in the temperatureammonium concentration plane. $\mathrm{FE}$ denotes the ferroelectric region, $\mathrm{PE}$ denotes the paraelectric region, F-P denotes the mixed ferroelectric and paraelectric region, F-G denotes the mixed ferroelectric and proton glass region and PG denotes the proton glass region. 
This work was support by National Science Foundation Grant DMR-9017429.

\section{REFERENCES}

[1] N. J. Pinto, K. Ravindran and V. H. Schmidt, "Field-heated, field-cooled, and zero-field-heated static permittivity of the deuteron glass $\mathrm{Rb}_{1-\mathrm{x}}\left(\mathrm{ND}_{4}\right)_{\mathrm{X}} \mathrm{D}_{2} \mathrm{AsO}_{4}$," Phys. Rev. $\mathrm{B}$, vol. 48 , pp. 3090-3094, August 1993.

[2] A. K. Jonscher, Dielectric Relaxation in Solids. London: Chelsea Dielectrics Press, 1983, ch. 5, p. 234.

[3] Shane Stadler, The Dielectric Properties of the Proton Glass $\mathrm{Rb}_{1-x}\left(\mathrm{NH}_{4}\right)_{x} \mathrm{H}_{2} \mathrm{PO}_{4}$. M. S. Thesis, University of North Dakota, 1992 , p. 46.

[4] M. Takashige, H. Teruchi, Y. Miura and S. Hoshino, "A ReEntrant Glasslike Phase in $\mathrm{Rb}_{1-\mathrm{x}}\left(\mathrm{NH}_{4}\right)_{\mathrm{X}} \mathrm{H}_{2} \mathrm{PO}_{4}$," Jour. Phys. Soc. Japan, vol. 54, pp. 3250-3253, 1985.

[5] A.A. Volkov, G. V. Kozlov, S.P. Lebedev, A.V. Sinitski, S. Kamba, and J. Petzelt, "Soft Modes in RADP Glass," Soviet Phys. JETP, vol. 74, pp. 133-136, 1992.

[6] Ref. [3], p. 36.

[7] Ref. [3], pp. 47-49.

[8] G. Papantopoulos, G. Papavassiliou, F. Milia, V. H. Schmidt, J. E. Drumheller, N. J. Pinto, R. Blinc and B. Zalar, "75 As Nuclear Quadrupole Resonance in Weakly Substitutionally Disordered $\mathrm{Rb}_{1-\mathrm{x}}\left(\mathrm{NH}_{4}\right)_{\mathbf{x}} \mathrm{H}_{2} \mathrm{AsO}_{4}$," Phys. Rev. Lett., vol. 73, pp. 276-279, July 1994 .

[9] Ref. [3], p. 52.

[10] A. Heuer and R. J. Silbey, "Tunneling in real structural glasses: a universal theory," Phys. Rev. B, vol. 49, pp. 1441-1444, January 1994. 